\title{
Multiresolution analysis of solar mm-wave bursts
}

\author{
U. Schwarz ${ }^{1}$, J. Kurths ${ }^{1}$, B. Kliem ${ }^{2}$, A. Krüger ${ }^{2}$, and S. Urpo ${ }^{3}$ \\ 1 Institut für Theoretische Physik und Astrophysik, Universität Potsdam, Am Neuen Palais, D-14469 Potsdam, Germany \\ 2 Astrophysical Institute Potsdam, An der Sternwarte 16, D-14482 Potsdam, Germany \\ 3 Helsinki University of Technology, Otakaari 5a, SF-02150 Espoo, Finland
}

Received October 28, 1996; accepted May 13, 1997

\begin{abstract}
Two methods of multi-scale time series analysis are applied to solar mm-wavelength flux time profiles in order to assess the diagnostic power of these tools for the exploration of nonlinear energy release processes. Both the multiresolution analysis (MRA), a method based on the wavelet transform, and the structure function analysis (SFA) permit the treatment of non-stationary time series. In addition, the MRA offers a local decomposition of the scaling behavior of the flux variations. Our main emphasis is directed at a decomposition of the contributions of the different time scales to the overall flux profile. The methods yield consistent values of the "spectral index" which describes the scalings contained in the time series.

We find that time profiles of bursts are qualitatively analogous to fractional Brownian motion (fBm), possessing long-range temporal correlations. Such correlations are not found in quiet Sun observations.

The MRA of six solar mm-wave bursts shows that the radio flux is always composed of contributions from a broad range of time scales. Also during the main phase of bursts, which appears to be structurally analogous to the pre- and post-burst phases at a resolution limit of $1 \mathrm{~s}$, flux fluctuations are enhanced in a broad range of time scales. This suggests that the mm-wave bursts are composed of unresolved elements, just as the pre- and post-burst time profiles. The underlying energy release thus appears to be fragmentary. These results are discussed in terms of the avalanche model and plasma physical models for solar energy release events.
\end{abstract}

Key words: Sun: radio radiation — Sun: flares methods: data analysis

Send offprint requests to: U. Schwarz, USchwarz@agnld.unipotsdam.de

\section{Introduction}

Solar radio bursts are typical examples of superpositions of transient phenomena, i.e. the underlying physical processes are non-stationary (Isliker \& Kurths 1993). To study the energization and emission processes that cause bursts, data analysis methods are required which can be applied to such non-stationary time series. Promising methods of this kind are the structure function analysis (SFA) and the multiresolution analysis (MRA), whose potential in the investigation of such bursts is discussed here. In particular, the wavelet transform of the MRA permits a local decomposition of the scaling behavior in a time series - in contrast to methods designed to detect global properties, such as the Fourier analysis and the SFA.

Using these methods we look for structural differences between time series of the flux of different sources (bursts, quiet Sun, and sky background) as well as between different phases of the bursts at mm-wavelengths.

Statistical studies of solar energy release events, e.g. the distribution of event number versus energy content as observed at hard X-rays, have led to a description in terms of avalanches in a corona which has stored energy and is in a state of self-organized criticality ( $\mathrm{Lu} \&$ Hamilton 1991). The observed power-law distribution naturally follows from that model. One basic property of this model is that the system under consideration has no characteristic spatial scale above an elementary scale of the smallest avalanche (the smallest energy release event), up to the system size, the size of active regions. This elementary scale, which is a characteristic of the involved plasmaphysical process, is below the current resolution limit, since the power-law distribution extends down to the resolution limit. The success of this approach suggests that the energy release is basically fragmentary, the events being composed of elementary building blocks.

Many attempts have been made to resolve the elementary building blocks of the energy release and possibly also larger characteristic scales from the time profile of the solar flux, particularly in the radio and the hard $\mathrm{X}$-ray ranges. Most of these studies have concentrated on 
the shortest time scales detectable. For example, Kiplinger et al. (1983) have found fast hard X-ray spikes with duration down to $45 \mathrm{msec}$ and Güdel \& Benz (1990) have found similar durations in decimeter radio wavelength spike burst observations. Here we follow a different approach by attempting to resolve a broad range of time scales above $0.5 \mathrm{~s}$ present in a flux record of the emission at millimeter wavelengths. Thus, we quantify the general structure of the emission and study its evolution in time, and we are able to investigate whether a hierarchy of dominant time scales is present in the flux profile, as has been discussed previously (Krüger et al. 1987, 1994), or whether the distribution of contributing time scales is more or less structureless, as predicted by the avalanche model. In comparison to hard X-ray data, the choice of this wavelength range has the advantage that the flux profile before and after bursts can also be analyzed and compared with the burst data (since detector or sky noise contributions remain small), and in comparison to the decimetric range the mm-wavelength flux profile is supposed to reflect the energy release process in a more direct manner, less influenced by nonlinear plasma processes which may modulate the radio wave intensity.

The organization of this paper is as follows: In the following section we present the data. In Sect. 3 we introduce the tools of data analysis: SFA and MRA. The diagnostic capabilities of these tools in the application to the radio burst time profiles are discussed in Sect. 4. In Sect. 5, we discuss the physical interpretation of some features of the obtained results, and Sect. 6 presents the conclusions.

\section{The microwave data}

We analyze several microwave bursts digitally observed at $36.8 \mathrm{GHz}(8.2 \mathrm{~mm}$ wavelength) at the Metsähovi Radio Research Station of the University of Technology Helsinki/Finland (cf. Table 1). The receiver is connected with a Cassegrain telescope of $14 \mathrm{~m}$ diameter resulting in a half-power beam width of 2.4 arcmin at this frequency. The quiet-Sun level at this frequency is $7800 \mathrm{~K}$ (Urpo et al. 1992). Typical time profiles are shown in Figs. 1-3. Each data set has a duration of about one hour with a time resolution of $0.5 \mathrm{~s}$.

The analyzed bursts originated in AR 6555 (Solar Geophysical Data). The observations are chosen because they show variations in a rather broad range of time scales. In particular the events of Figs. 1 and 2 exhibit shortperiod radio spikes which are known to appear frequently at dm-waves but are rare at solar bursts in the short cm- and mm-wave ranges (Slottje 1978; Benz 1986; Benz et al. 1992). The event shown in Fig. 3 appears to possess a different temporal dynamics than the other two bursts. Moreover, the temporal structure of the pre-burst flux profile in Fig. 1 appears to differ from that of the post-burst profile. This raises the question whether the structure of

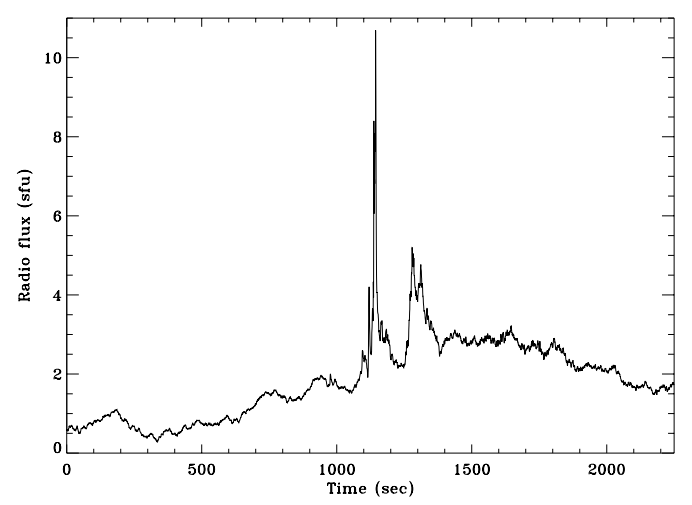

Fig. 1. Radio flux time profile of a burst at $36.8 \mathrm{GHz}$ observed on March 23, 1991 at the Metsähovi Radio Research Station of the Helsinki University of Technology. A section of the event starting at 12:12 UT is shown

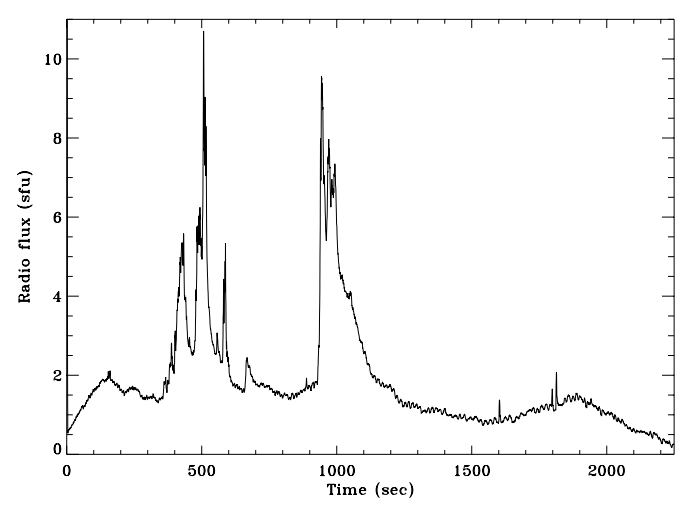

Fig. 2. Radio flux time profile of a burst at $36.8 \mathrm{GHz}$ observed on March 24, 1991, 10:00 UT

those diverse flux profiles can be quantitatively described in a uniform manner.

For later comparison, we add here in Fig. 4 the time profile of a synthetic realization of a fractional Brownian motion (fBm) process with a spectral index similar to that of the radio flux profile shown in Fig. 3 .

\section{Data analysis}

In a first attempt to analyze the burst, quiet Sun, and sky time profiles, sliding power spectra were used, but this did not reveal clear-cut patterns. The power spectra calculated from different time intervals have quite different shapes, i.e., they depend on time essentially. Therefore, we check whether the SFA and the MRA are more convenient to describe these non-stationary burst time profiles (Kurths et al. 1995). It is important to note that no filtering is required to apply these methods. 
Table 1. Scaling exponents calculated by Eq. (2) and spectral indices of the analyzed mm-wave bursts. Spectral indices $\alpha_{\mathrm{SF}}$ are calculated by Eq. (6), and $\alpha_{\mathrm{MRA}}$ are determined from the scalegrams (Figs. 11-13, 15-17). We omitted the values of $H_{\mathrm{SF}}$ and $\alpha_{S F}$ for the main burst phases because their scatter becomes exceedingly large in such short intervals

\begin{tabular}{|c|c|c|c|c|c|}
\hline Date & $\begin{array}{c}\text { Start } \\
\mathrm{UT}\end{array}$ & $\begin{array}{c}\text { Duration } \\
(\mathrm{min})\end{array}$ & $\begin{array}{c}\text { Scaling exponent } \\
H_{\mathrm{SF}}\end{array}$ & $\begin{array}{c}\text { Spectral index (SF) } \\
\alpha_{\mathrm{SF}}\end{array}$ & $\begin{array}{c}\text { Spectral index (MRA) } \\
\alpha_{\mathrm{MRA}}\end{array}$ \\
\hline \hline March 23, 1991 (Fig. 1) & $11: 58$ & 58.5 & $0.57 \pm 0.1$ & $2.13 \pm 0.2$ & $2.21 \pm 0.13$ \\
Pre burst phase & $11: 58$ & 31.5 & $0.66 \pm 0.04$ & $2.32 \pm 0.08$ & $2.15 \pm 0.28$ \\
Main phase & $12: 29$ & 6.0 & & & $2.24 \pm 0.13$ \\
Post burst phase & $12: 35$ & 21.0 & $0.45 \pm 0.08$ & $1.90 \pm 0.15$ & $1.91 \pm 0.08$ \\
\hline March 23, 1991 (Fig. 3) & $12: 58$ & 47.5 & $0.66 \pm 0.06$ & $2.31 \pm 0.12$ & $2.29 \pm 0.14$ \\
\hline March 23, 1991 & $14: 00$ & 61.1 & $0.57 \pm 0.04$ & $2.13 \pm 0.08$ & $2.21 \pm 0.14$ \\
\hline March 24, 1991 (Fig. 2) & $10: 00$ & 63.1 & $0.58 \pm 0.13$ & $2.15 \pm 0.26$ & $1.99 \pm 0.11$ \\
Pre burst phase & $10: 00$ & 5.1 & $0.44 \pm 0.05$ & $1.87 \pm 0.1$ & $1.96 \pm 0.11$ \\
Main phase & $10: 05$ & 14.0 & & & $2.37 \pm 0.11$ \\
Post burst phase & $10: 19$ & 44.0 & $0.41 \pm 0.05$ & $1.82 \pm 0.1$ & $1.90 \pm 0.36$ \\
\hline March 24, 1991 & $11: 09$ & 58.3 & $0.49 \pm 0.25$ & $1.92 \pm 0.5$ & $2.04 \pm 0.15$ \\
Pre burst phase & $11: 09$ & 7.0 & $0.39 \pm 0.03$ & $1.77 \pm 0.05$ & $1.77 \pm 0.56$ \\
Main phase & $11: 16$ & 2.5 & & & $2.71 \pm 0.16$ \\
Post burst phase & $11: 18$ & 48.8 & $0.25 \pm 0.04$ & $1.49 \pm 0.08$ & $1.55 \pm 0.59$ \\
\hline March 24, 1991 & $13: 41$ & 91.0 & $0.52 \pm 0.15$ & $2.04 \pm 0.3$ & $2.14 \pm 0.62$ \\
Pre burst phase & $13: 41$ & 28.0 & $0.49 \pm 0.04$ & $1.98 \pm 0.08$ & $1.79 \pm 0.61$ \\
Main phase & $14: 09$ & 20.0 & & & $2.15 \pm 0.63$ \\
Post burst phase & $14: 29$ & 43.0 & $0.47 \pm 0.04$ & $1.93 \pm 0.08$ & $2.14 \pm 0.31$ \\
\hline August 2, 93: Quiet Sun & $9: 58$ & 17.8 & $0.22 \pm 0.02$ & $1.44 \pm 0.04$ & $1.37 \pm 0.59$ \\
\hline August 2, 93: Sky & $10: 42$ & 21.8 & $0.14 \pm 0.02$ & $1.27 \pm 0.04$ & $1.03 \pm 0.96$ \\
\hline
\end{tabular}

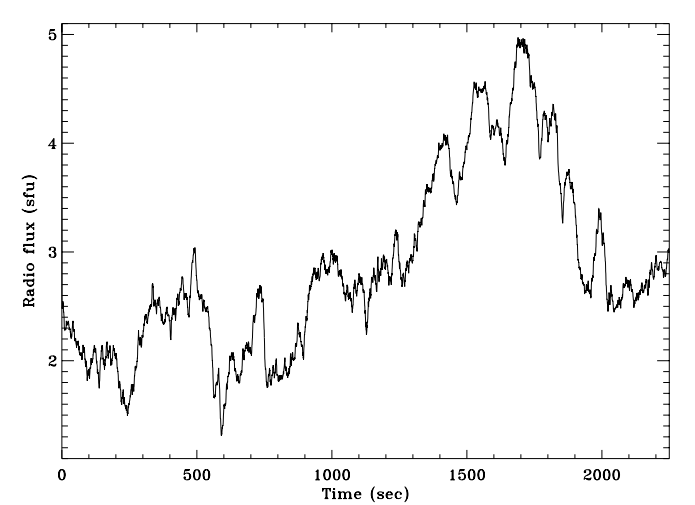

Fig. 3. Radio flux time profile of a burst at $36.8 \mathrm{GHz}$ observed on March 23, 1991, 12:58 UT. The flux time profiles of the preand post-burst phases of the events presented in Figs. 1 and 2 look very similar to this one

\subsection{Structure function}

The concept of self-affinity presented in the following is a promising approach to describe a uniform broad-range scaling in time, although the underlying process can be non-stationary (Feder 1988). This concept is a generalization of self-similarity, which is the basis of (deterministic) fractal geometry. A convenient way to quantify self-affinity

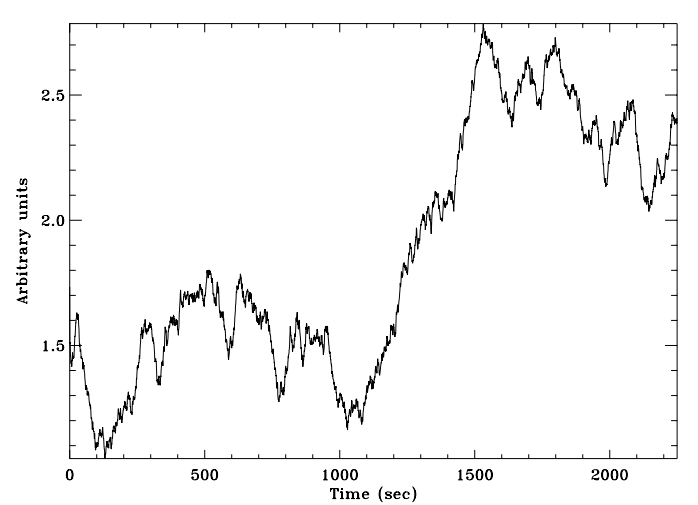

Fig. 4. Realization of fractional Brownian motion with a spectral index $\alpha=2.25$. This signal resembles well the observed radio flux shown in Fig. 3 and zoomed radio fluxes of the preand post-burst phases of the events shown in Figs. 1 and 2

is based on the structure function ( $\mathrm{SF}$; two-point correlation function)

$S(\lambda)=\left\langle|x(t+\lambda \Delta t)-x(t)|^{2}\right\rangle_{t}$

where $\langle\ldots\rangle_{t}$ is the average over time $t$, and $\lambda$ takes integer values. $\Delta t$ denotes the sampling time and $x(t)$ is the observed time series. If a process is self-affine then this SF obeys the following power-law scaling with $\lambda$

$S(\lambda)=\lambda^{2 H} S(1)$ 


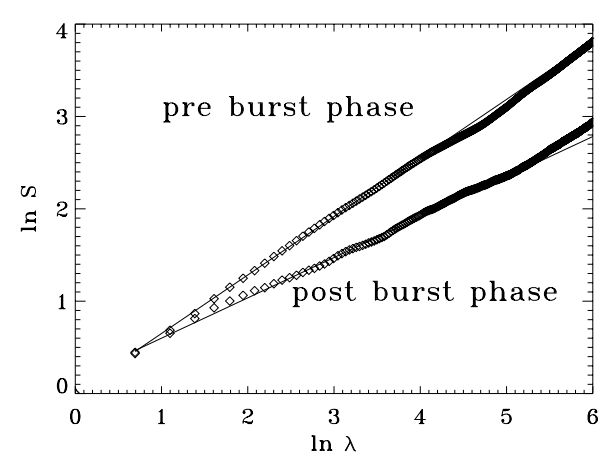

Fig. 5. Double logarithmic plot of the structure function $S(\lambda)$ estimated (diamonds) from the radio flux of the event presented in Fig. 1. Thin solid lines show the linear fits used to derive the scaling exponents $H_{\mathrm{SF}}$

where $H$ is the characteristic scaling exponent. Selfaffinity means that a scaling exponent $H$ does exist independently of the shift $\lambda$ in time. A typical example of a self-affine process is the fractional Brownian motion $(0<H<1)$ which generalizes the classical Brownian motion or random walk, where $H=0.5$ (Feder 1988). Such a process was investigated by Osborne \& Provenzale (1989). Starting from a power-law decay of its power spectrum $P\left(\omega_{k}\right)$,

$P\left(\omega_{k}\right)=C \omega_{k}^{-\alpha}$,

with spectral index $\alpha$, they construct a stochastic time series by

$x_{i}=\sum_{k=1}^{N / 2} \zeta_{k} \cos \left(\omega_{k} i \Delta t+\varphi_{k}\right), \quad i=1, \ldots, N$,

where $\omega_{k}=k \Delta \omega(k=1, \ldots, N / 2)$, with $\Delta \omega=2 \pi / N \Delta t$, and $\zeta_{k}=\sqrt{P\left(\omega_{k}\right) \Delta \omega}$. The $\varphi_{k} \in[0,2 \pi)$ were chosen at random. Such $\mathrm{fBm}$ is characterized by a power-law scaling in time (Eq. 2) as well as in frequency

$P(f) \propto f^{-\alpha}$.

Both scaling exponents are simply related by

$H=\frac{\alpha-1}{2}$.

It is important to note that the $\mathrm{fBm}$ process is not stationary because the standard deviation $\sigma_{\mathrm{X}}$ of the time series depends on the length of the time interval $\tau$ in which it is calculated. This standard deviation also scales as a powerlaw

$\sigma_{\mathrm{X}}(\tau) \propto \tau^{a}$

Therefore, fBm cannot be characterized by the correlation dimension. The main difference to self-similar processes is that $\mathrm{fBm}$ exhibits a different scaling in space and time. The concept of fractal dimensions requires, however, that both scalings are identical.

For $H=0.5$ in Eq. (2), we have the classical Brownian motion, i.e. the increments are not correlated. In the case $H>0.5$, there is a positive persistence. Therefore, an increasing trend in the past implies an increasing trend in the future, i.e. processes with $H>0.5$ are characterized by long-range correlations.

Since the analyzed microwave bursts are far from being stationary, we calculate the $\mathrm{SF}, S(\lambda)$ (Eq. 1), of the radio flux time profiles. Surprisingly, for each event we find a well-expressed power-law scaling (cf. Fig. 5 and Table 1) in a rather broad region of $\lambda$. It turns out that two of the analyzed events are compatible with the usual Brownian motion. The other ones are characterized by scaling exponents significantly larger than 0.5 which is typical for $\mathrm{fBm}$ with rather long-range correlations (Table 1 ).

If Eq. (2) is applied to white noise, $H=0$ is obtained. In fact, the quiet Sun and sky observations yield $H$ which approach that value (Table 1 ).

The power-law scaling of the SF of the radio flux as well as zooming of the time profile suggest that there is no dominating narrow-band time scale. It is important to note that the SF, like the power spectrum, yields only global properties of the temporal behavior of the radio flux. In the following we present the MRA which is more suitable to quantify local and time-dependent phenomena, which are typical of solar radio bursts.

\subsection{Multiresolution analysis}

In the general case of non-stationarity, we have to expect an inhomogeneous scaling behavior, varying in space and/or in time. Detailed information on both, the location and the size of the characteristic features is of interest here. Wavelets are a proper tool to analyze such phenomena. It should be mentioned that the well-known windowed Fourier transform is another tool to study local behavior, but it is a much more coarse-grained one (cf. Daubechies 1992; Scargle 1993; Scargle et al. 1993).

We first recall a few basics about the most common global technique, the power spectrum, which is based on the Fourier transform (Priestley 1981). It is an efficient tool for giving some dominant frequencies (or characteristic sizes). This transform is a projection on an orthogonal basis consisting of harmonic functions. Hence, there exists a unique decomposition and reconstruction formula for a given function $x(t)$, but there is no simple relationship between the local behavior of $x(t)$ and the Fourier coefficients. This information is so deeply buried in the phases of the coefficients that it is very difficult to retrieve.

In generalization, the notion of wavelet analysis addresses both, unknown periodicities and non-stationary structures. The wavelet analysis is based on time-limited elements, the wavelets. By this means, one has the 


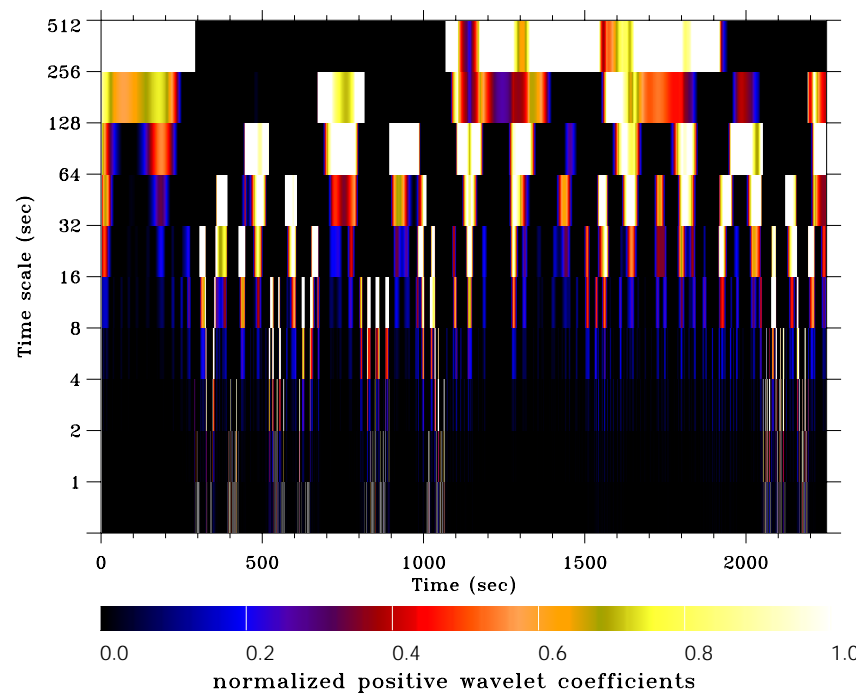

Fig. 6. Scalogram of the event presented in Fig. 1. We have plotted the normalized positive wavelet coefficients. White pattern represent dominant time scales. The dark background represents wavelet coefficients with very small amplitudes. For clarity, 5 sec averages are plotted

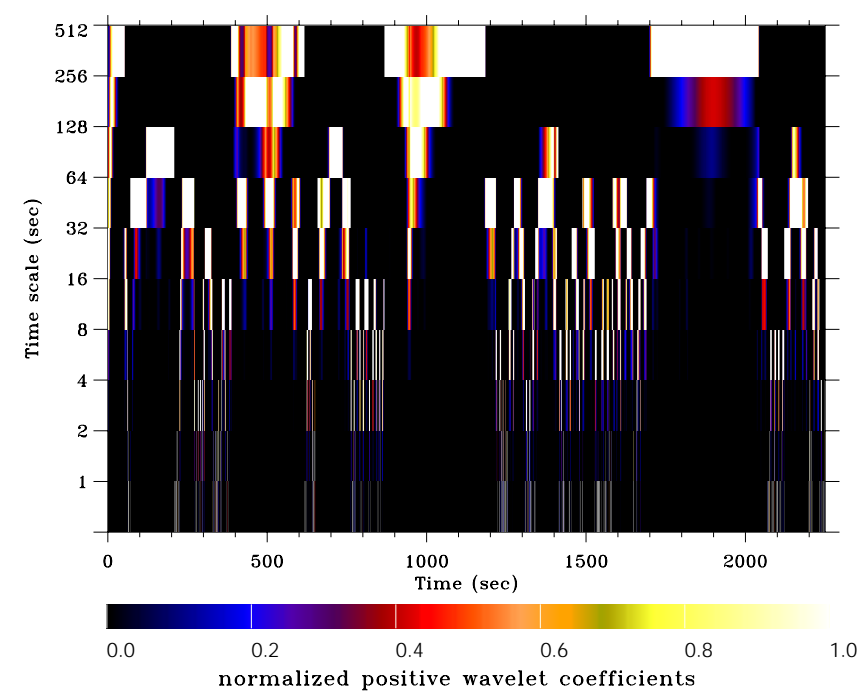

Fig. 7. Scalogram of the event presented in Fig. 2

possibility of dealing with non-stationary time series, where, e.g., some coherent structures evolve in time. The wavelet transform of $x(t)$ is the decomposition into a basis of functions $w_{a, b}(t)$ with

$$
w_{a, b}(t)=|a|^{-1 / 2} w\left(a^{-1}(t-b)\right),
$$

all derived from a unique function $w(t)$, called the "mother wavelet," by translation $b$ and scaling $a$. Several functions have been recommended as wavelets, e.g., Daubechies wavelets and Gabor-Malvar wavelets. For our purpose, the simple triangle-like wavelets (cf. Mallat 1989; Meyer \& Ryan 1993; Vigouroux \& Delache 1994) are appropriate,

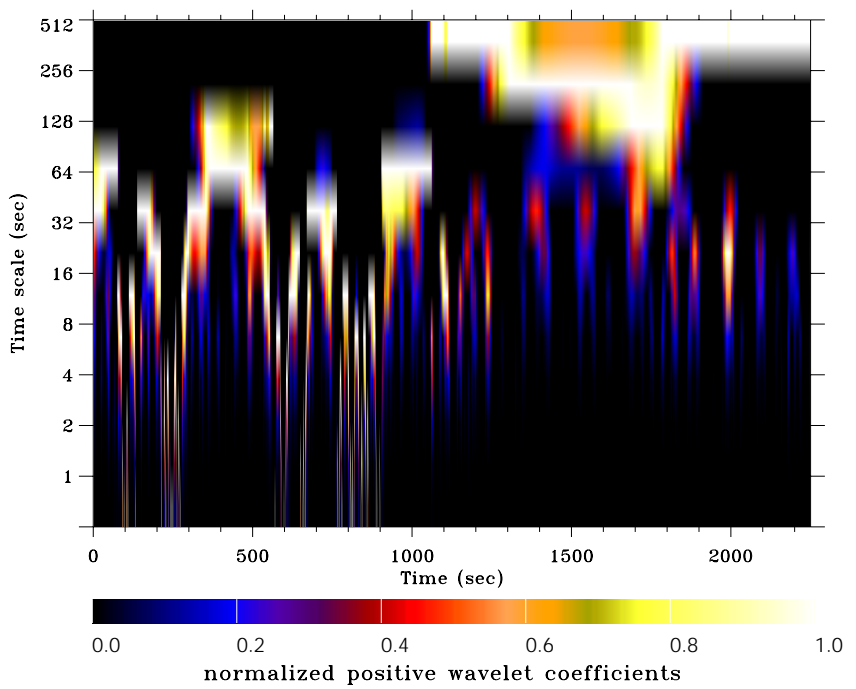

Fig. 8. Scalogram of the event presented in Fig. 3

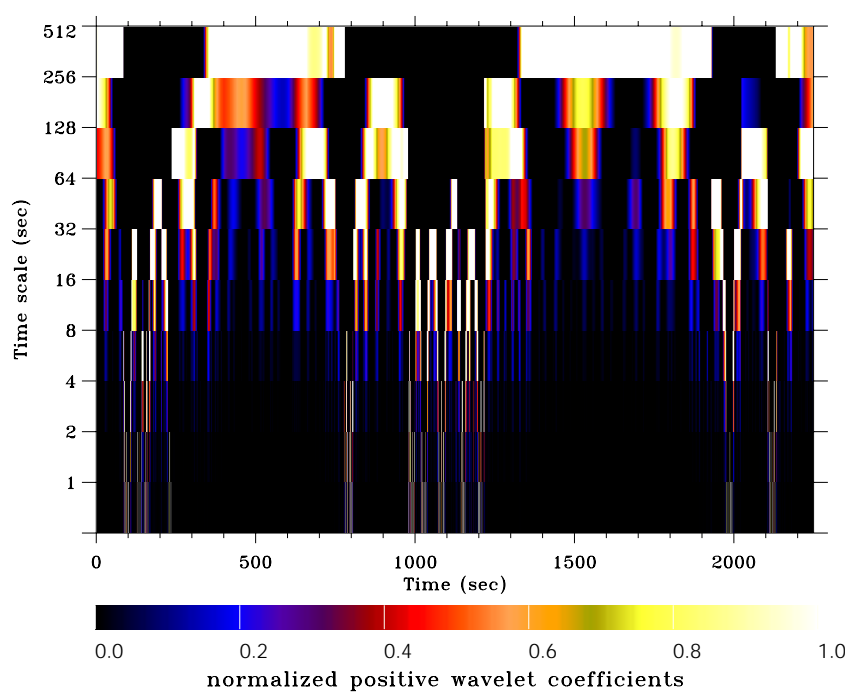

Fig. 9. Scalogram of a $\mathrm{fBm}$ realization with $\alpha=2.25$ presented in Fig. 4

because their shape fits the time profile of the radio flux quite well.

We use a bi-orthogonal wavelet basis consisting of the collection $w_{j, k}(t)$, where $j \in N$ is the scaling factor and $k \in Z$ (set of relative integers) is the translation, together with $q(t-k), k \in Z$, where $q(t)$ is a smooth function with a rapid decay. On this basis, $x(t)$ can uniquely be written as

$x(t)=\sum_{k \in Z} c_{k} q(t-k)+\sum_{j \in N} \sum_{k \in Z} d_{j, k} w_{j, k}(t)$. 

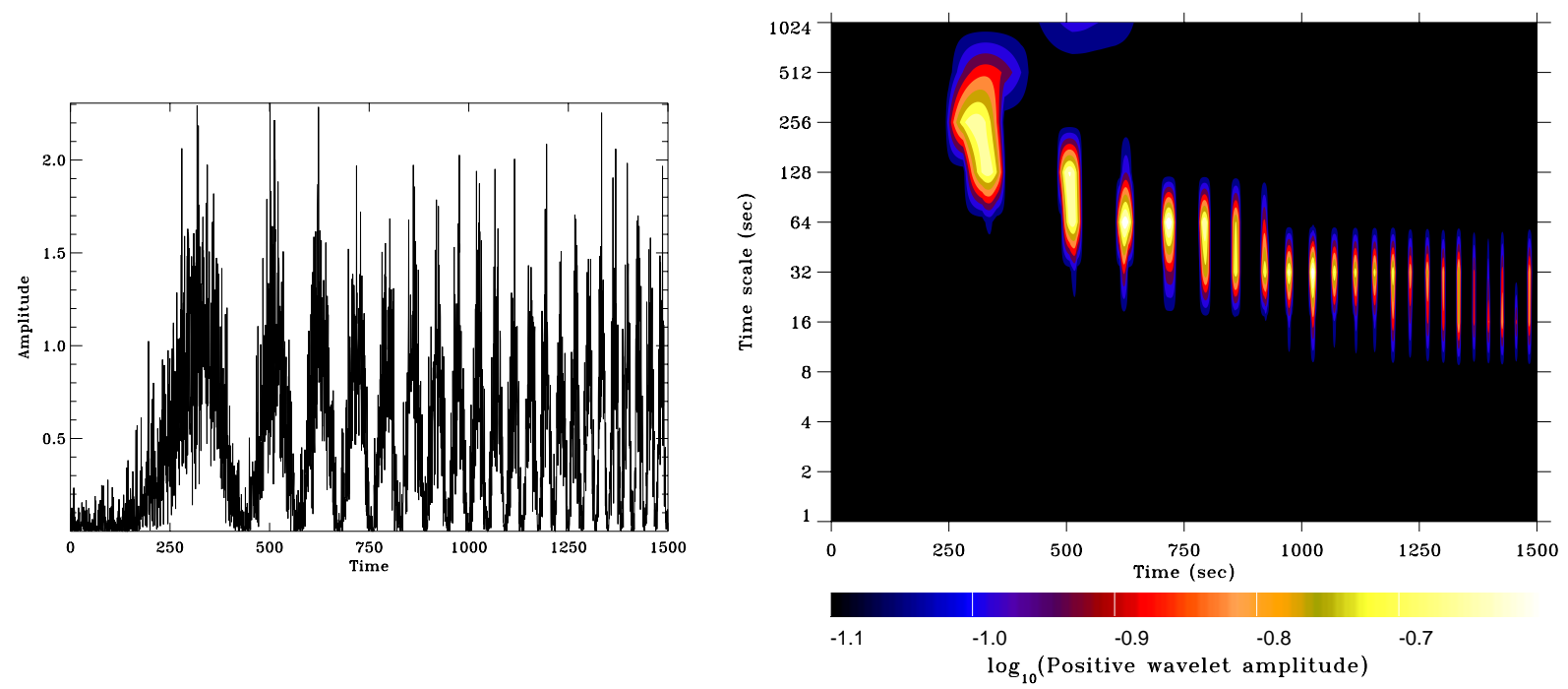

Fig. 10. Illustration of the MRA. Left: Wake-like data according Eq. (14). Right: Scalogram. The scalogram shows clearly both the location and the width of the dominant features of the data

The two functions $q(t)$ and $w(t)$ cannot be chosen independently. Among the many possibilities for the two functions $q(t)$ and $w(t)$, we choose, following Bendjoya et al. (1993),

$q(t)=\left\{\begin{array}{cl}1-|t| & \text { for }|t| \leq 1 \\ 0 & \text { otherwise }\end{array}\right.$

and

$w(t)=\left\{\begin{array}{cl}\frac{1}{4}|t|-\frac{1}{2} & \text { for } \quad 1<|t| \leq 2, \\ \frac{1}{2}-\frac{3}{4}|t| & \text { for } \quad|t| \leq 1 \\ 0 & \text { otherwise. }\end{array}\right.$

A main advantage of wavelets is the possibility to calculate the coefficients $c_{k}^{j}$ and $d_{k}^{j}$ recursively. The first trivial step is to take the function $q(t)$ with the same resolution as the sampled signal. We only have to compute the $c_{k}^{0}$ (the superscript refers to the number of the step in the iterative process). From the formula for $q(t)$, it is easy to see that the $c_{k}^{0}$ are simply the sampled values of $x(t)$.

Then we scale the resolution. We choose the normalizing coefficients such that we replace $q(t)$ by $q(t / 2) / 2$. There is only one level of fine fluctuations to add to the smoothed part to recover the signal, and it is defined by the set of $d_{1, k}=d_{k}^{1}$. We can now repeat the same procedure to the smoothed part of level 1 . This gives two new sets of coefficients $c_{k}^{2}$ and $d_{k}^{2}$.

With our choice of $q(t)$ and $w(t)$, the formulae to obtain the $c_{k}^{j}$ and the $d_{k}^{j}$ are simple recursions (Mallat 1989):

$c_{k}^{j}=\frac{1}{2} c_{k}^{j-1}+\frac{1}{4}\left(c_{k-2^{j-1}}^{j-1}+c_{k+2^{j-1}}^{j-1}\right), \quad j \geq 1$,

and

$d_{k}^{j}=c_{k}^{j-1}-c_{k}^{j}$.
These equations can easily be interpreted in terms of filtering. The $c_{k}^{j}$ are obtained by applying a low-pass filter to the $c_{k}^{j-1}$ (Eq. 12). On the other hand, the $d_{k}^{j}$ being obtained by a difference between two levels of $c_{k}^{j}$ are in fact the result of a band-pass filter applied to the signal (Eq. 13). This algorithmic scheme is called time-scale analysis or multiresolution analysis (MRA).

The decomposition described above is best portrayed in a $2^{j} \Delta t-k \Delta t$ - plot of the coefficients $d_{k}^{j}$, called scalogram (Figs. 6-9), which shows the scaling behavior of the radio flux $x(t)$ in dependence on the time location $k \Delta t$. Different intensities of the coefficients (amplitudes) are displayed by different grey levels. The computational effort of the chosen recursive procedure is similar to that for calculating the fast Fourier transform.

To illustrate the MRA, we take the following function (Fig. 10)

$x(t)=\sin ^{2}\left(\frac{t}{275}\right)^{\frac{5}{2}}$

and distorted it somewhat by adding noise. Such a time series is non-stationary, but its characteristics are displayed quite well by the scalogram (Fig. 10).

\section{Scalograms and scalegrams of radio fluxes}

Next, we apply the MRA to the microwave burst events listed in Table 1. The problem we are interested in is a description of a broad band of time scales in microwave bursts, in order to support the diagnostics of the underlying coronal energy release processes (Krüger et al. 1994).

Using the functions given by Eqs. (10) and (11), we observe that the positive wavelet coefficients $d_{k}^{j}$ reflect the burst-like behavior of the radio flux quite well. In 


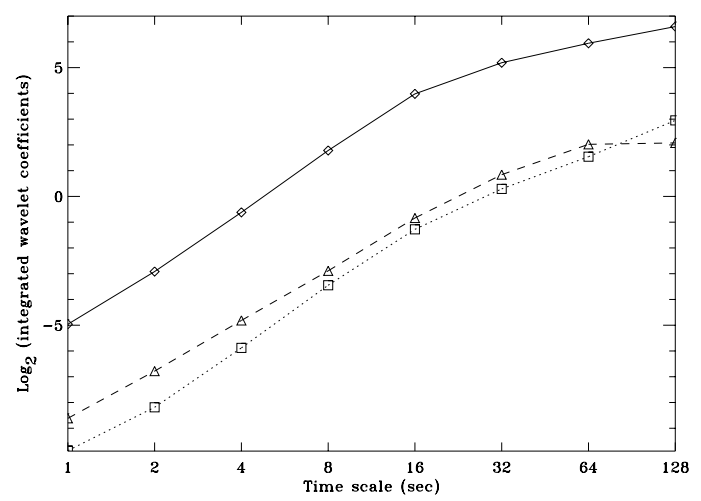

Fig. 11. Scalegrams for different time intervals of the event presented in Fig. 1. Pre-burst phase (dotted line; squares): $0-1000 \mathrm{~s}$, main phase (solid line; diamonds): $1000-1500 \mathrm{~s}$, post-burst phase (dashed line; triangles): $1500-2500 \mathrm{~s}$

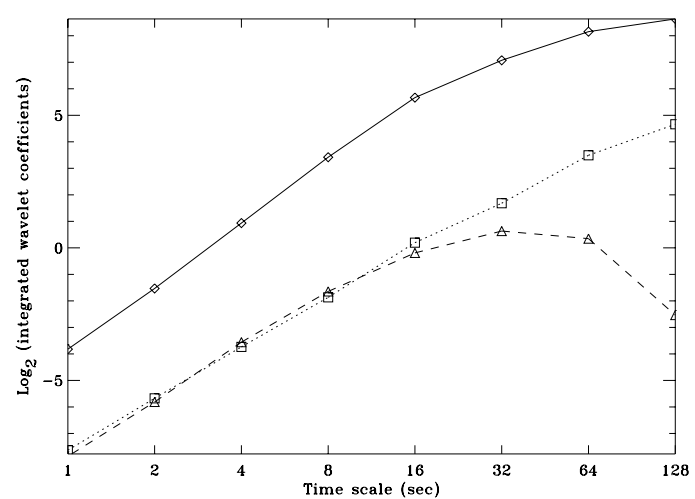

Fig. 12. Scalegrams of the event presented in Fig. 2. Dotted line (squares): $0-350 \mathrm{~s}$, solid line (diamonds): $350-1300 \mathrm{~s}$, dashed line (triangles): $1500-1800 \mathrm{~s}$

Figs. 6-9 the logarithm of the normalized positive wavelet coefficients $\log _{2} d_{k}^{j}$ is plotted (scalogram). Summing up the wavelet coefficients over time (index $k$ ), we obtain a picture similar to a power spectrum, the so-called scalegram (Figs. 11-14):

$s(j)=\left\langle\left|d_{k}^{j}\right|^{2}\right\rangle_{k \in \text { time interval }}$

This allows to calculate the spectral index $\alpha_{\mathrm{MRA}}$ from the slope of that part of the scalegram which follows a power law (Flandrin 1994). Figure 14 shows that, for a process with structural similarity to $\mathrm{fBm}$, the lengths of the available time series permit to derive the scaling up to about $16 \mathrm{~s}$. The observations in fact show a scaling (power law behavior of the scalegram) from the limit set by the resolution $(1 \mathrm{~s})$ up to $\sim 16 \mathrm{~s}$ (Figs. 11-13) with minor deviations for the shortest data sets $(<500$ data points). For this range the spectral indices $\alpha_{\mathrm{MRA}}$ have been estimated; they are shown in Figs. 15-18 and listed

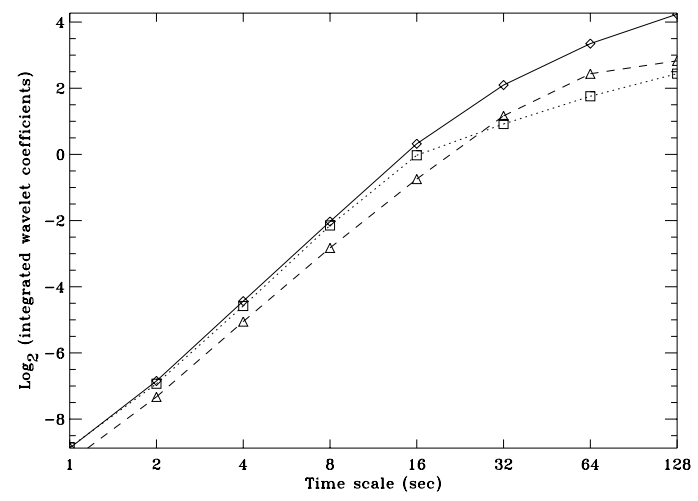

Fig. 13. Scalegrams of the event presented in Fig. 3. Dotted line (squares): $0-500 \mathrm{~s}$, solid line (diamonds): $800-1800 \mathrm{~s}$, dashed line (triangles): $1800-2200 \mathrm{~s}$

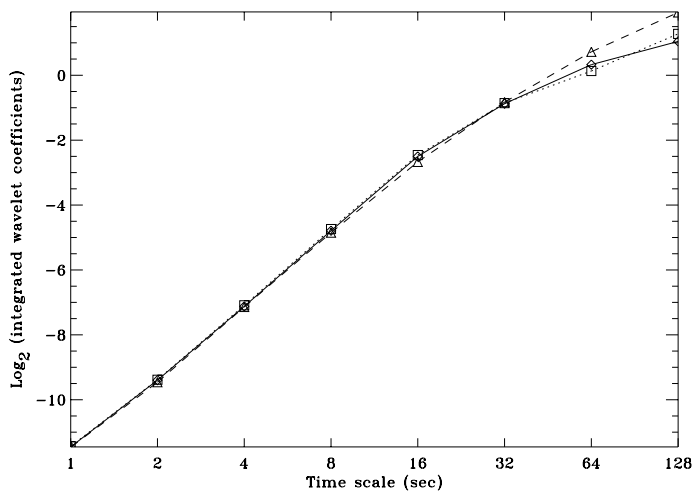

Fig. 14. Scalegrams of a fBm realization with $\alpha=2.25$ presented in Fig. 4. The three different scalegrams are calculated for the same intervals as in Fig. 13

in the last column of Table 1 . They agree quite well with the spectral indices calculated from the structure function.

The wavelet transform calculated from the data indicates that a rather broad range of time scales from $1 \mathrm{~s}$ to a few minutes is involved during most parts of the events (Figs. 6-8, 11-13). The sequences of bright patterns in Figs. 6-8 give an impression of the dominant scales at different times during the bursts. Indications for hierarchic time structures (where different, well separated time scales become dominant) are only very weak and short-lived. For example, there are short periods around $t=1150 \mathrm{~s}$ and $t=1300 \mathrm{~s}$ in Fig. 6 and around $t=500 \mathrm{~s}$ in Fig. 7 where two maxima of the wavelet coefficients do exist.

For purposes of comparison we have also calculated the scalograms for several kinds of surrogate data, such as white noise, linear colored noise (autoregressive processes), and $\mathrm{fBm}$, using comparable data lengths. As expected, for white noise the scalogram exhibits complete disorder, whereas periodic features are transformed into a simple vertical stripe pattern. These stripes are located 


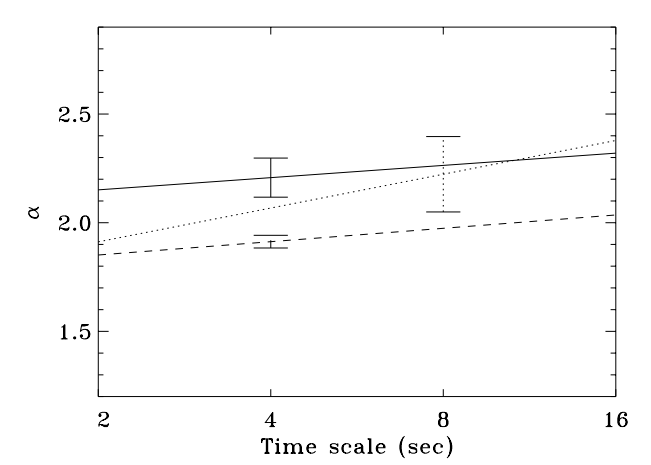

Fig. 15. Linear fits to the slopes in the power law regions of the scalegrams presented in Fig. 11. Error bars associated with these fits are shown and also given in the last column of Table 1

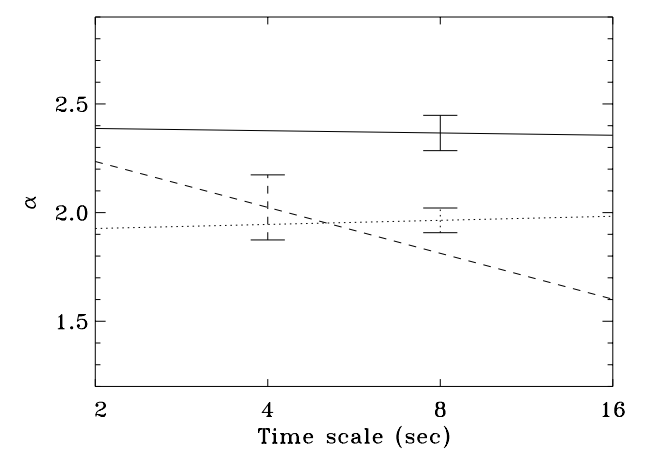

Fig. 16. Same as above for Fig. 12

at the maxima of the periodic signal. The vertical length of the stripes corresponds to the half width of the period length (see also Fig. 10).

If we compare the scalogram plots of these different models with the data, we find that the scalogram of an fBm with $H=0.625$ (Fig. 9) looks very similar to that of the bursts. This agreement is strengthened by the spectral index $\alpha_{\mathrm{MRA}}$ calculated from the slope of the scalegram, which nearly equals $\alpha_{\mathrm{SF}}=2.25$.

It is important to note that the scalograms from an off-Sun position and from a quiet region on the Sun differ significantly from those of the bursts. The corresponding spectral indices, given in Table 1, show that long-range correlations are missing in these time series $(H<0.5)$.

Next, we analyze the different phases of the burst separately, i.e. the spectral index $\alpha_{\mathrm{MRA}}$ is derived for the pre-impulsive phase, the main phase, and postimpulsive phase of the bursts shown in Figs. 1-3 separately. Surprisingly, we find that the spectral indices $\alpha_{\mathrm{MRA}}$ change only slightly between these intervals. Thus the three phases appear to be structurally analogous, i.e., the relative contribution of the different time scales remains roughly constant. There is a tendency for $\alpha$ to be slightly

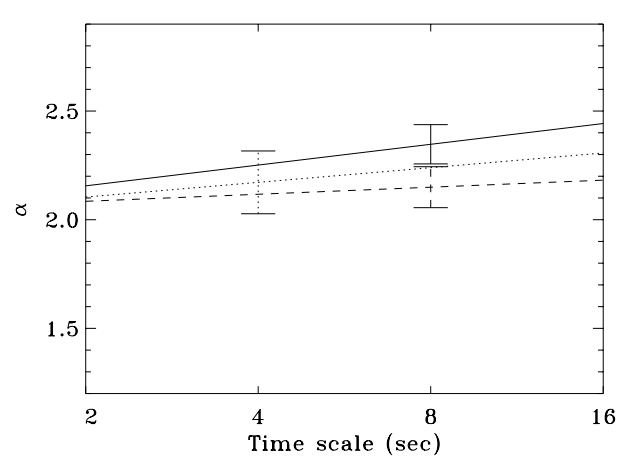

Fig. 17. Same as above for Fig. 13

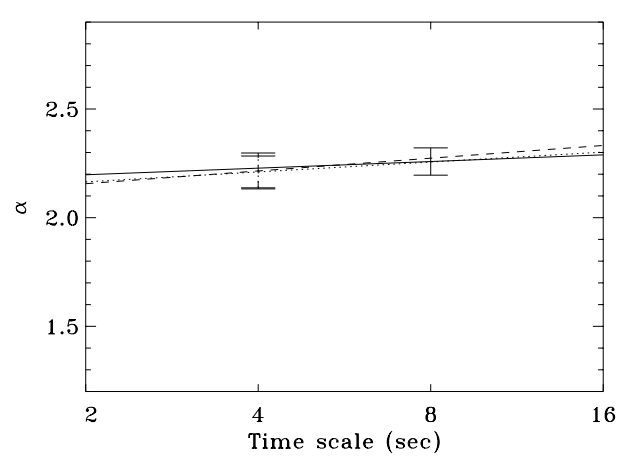

Fig. 18. Same as above for the $\mathrm{fBm}$ realization presented in Fig. 14

smaller in the post-impulsive phase, indicating that the emission becomes more random (less correlated) in that phase (Figs. 15-17).

In order to check the reliability of the scalegrams, we have calculated them in each case for 40 slightly different time intervals of equal length. We observe in all cases that the used method is rather robust. The error bars obtained from these sets of 40 scalegrams for each analyzed time interval are comparable to the symbol size in Figs. 11-14.

\section{Energy release models}

We discuss the main findings of our preceding data analysis in terms of models for impulsive energy releases in the solar corona. For an overview of the processes involved in the radio and related hard X-ray emissions from those energy release events see, e.g., Benz (1993), Benz \& Aschwanden (1992).

A general approach to energy release events in the solar corona is provided by the avalanche model $(\mathrm{Lu} \&$ Hamilton 1991; Lu et al. 1993; Vlahos et al. 1995). This statistical model supposes that there is an elementary building block of the energy release processes below the limit of resolution, possessing a threshold which depends 
only on local conditions, and that the corona is in a state of so-called self-organized criticality, i.e., everywhere and at all times close to onset of an energy release event. This state results from the interplay of external driving (energy input) and more or less localized energy release, where the energy release may organize itself into avalanches of all sizes, since every occurrence of the elementary building block may trigger further occurrences in neighboring regions due to spatial redistribution of stresses and free energy. Interpreting flare events as such avalanches of the elementary process, the model succeeded to reproduce the observed power-law distribution of the occurrence rate of flares versus energy content (Crosby et al. 1993). In order to obtain the power-law, one has to assume that energy release processes in the corona have no characteristic length scale greater than that of the elementary building block ( $\mathrm{Lu} \&$ Hamilton 1991). Then the occurrence rate of avalanches is a monotone decreasing function of energy content. Although the very nature of the energy release process is not addressed by this approach, it basically supposes that coronal energy release processes of all sizes have the same physical nature. This supposition is supported by our finding of structural similarity of preburst, burst, and post-burst phases, respectively. We suppose that the analyzed radio bursts are also composed of elementary building blocks (flux enhancements) not resolved by the available instrumentation, as suggested by their time profiles. One important difference between the scalegrams (Figs. 11-14) and the flare occurrence rate distribution needs to be clarified, however. While the latter is derived from a time series (several years long) in which every event occurs separately (i.e., localized in time), each of the former is derived from a time series of one single flare event, which is understood as a superposition of elementary energy release contributions, overlapping in time. Small-scale radio flux enhancements are then underrepresented in the scalograms and scalegrams, since in general they do not stand out clearly in the time profile of overlapping small-scale and large-scale flux enhancements.

The wavelet analysis and the structure function analysis both go beyond the description in terms of event occurrence rates by investigating the structure of single events. This refers to the question whether the energy release in one single flare or burst is composed of a number of avalanches, i.e., is fragmentary. If this is the case, it would be of interest whether the fragments possess a certain size, which would show up as a dominant time scale in the scalegrams. No signs of preferred avalanche sizes (maxima in the scalegrams) were found within the considered range of time scales, which again supports one of the basic assumptions of the avalanche model. We note, however, that there are indications of enhanced occurrence of $\sim 0.6 \mathrm{~s}$ spikes in hard X-ray time profiles of solar flares (Aschwanden et al. 1995). Work is currently underway to extend the time series analysis of radio bursts into this range of scales.
An understanding of the relative contribution of the various time scales to the total radio flux profile and possible evolutions of the structure of energy release events from the pre-burst to the main and post-burst phase can only be understood in terms of more detailed plasma physical models of the energy release process(es). We restrict ourselves to a brief reference to two currently discussed models, which have both been found consistent with observations of subsecond flux variations in solar bursts (Krüger et al. 1994), viz. the current sheet model and the electric circuit model. We remark that the current sheet model has recently found substantial observational support from X-ray observations of the Yohkoh satellite (Masuda et al. 1994; Tsuneta 1996).

The current sheet model involves the formation and dynamical evolution of current filaments at small spatial scales (Tajima et al. 1987; Kliem 1995). MHD simulations of current sheet instabilities at high magnetic Reynolds numbers show that the dynamical evolution leads to the pileup of gradients, i.e., the formation of smaller scales than initially present (Schumacher \& Kliem 1996). Since the electric field, which accelerates the particles, peaks in those regions, an enhancement of the small-scale contributions in the flux of fast particles (which is finally reflected by the radio flux) can be expected. Of course, the release of a large amount of energy during the burst phase (a large avalanche) requires the contribution from a large volume (an extended current sheet), hence large temporal scales will significantly contribute to the overall time profile if the release process involves a roughly uniform characteristic velocity (which is the Alfvén velocity).

The electric circuit model involves a combination of MHD and kinetic instabilities (Zaitsev \& Stepanov 1991, 1992) and, consequently, a broad range of time scales. Characteristically, $10-100 \mathrm{~s}$ scales are predicted by this model for subflares, which is roughly comparable to the maxima in the scalograms obtained here. A more thorough discussion of the two energy release models will become possible if the multiresolution analysis can be applied to burst data with a time resolution $\Delta t \ll 1 \mathrm{~s}$.

Finally we note that the reduced spectral index $\alpha$ in the post-burst phases in Figs. 11 and 12 is consistent with an important role of magnetohydrodynamic turbulence in the energy release process. After the main energy release, the energy in the turbulent motions cascades down to smaller scales where it is dissipated into heat, which shows up as a reduced slope in the scalegram.

\section{Conclusions}

We have studied the capabilities of two methods of nonlinear data analysis (multiresolution analysis and structure function analysis) to characterize complex, non-stationary time series, which are typically observed in solar microwave bursts. Our main findings are: 
(1) The wavelet transform permits a local decomposition of the scaling behavior of the temporal dynamics of the radio flux - in contrast to the usual global methods of data analysis based on the Fourier transform. This enables the study of non-stationary systems. Different time scales inherent in a series can be resolved, this can be done for different phases of the series separately. We have found that the occurrence of an impulsive component does not imply a significant structural change in the properties of scaling in time of the solar microwave flux. The scalegrams, obtained by averaging over the whole lifetime or a substantial fraction of a burst, do not show the dominance of a particular time scale in the considered range between $1 \mathrm{~s}$ and $\sim 2 \mathrm{~min}$; distinct hierarchic time structures and sequences of particular time scales are only short-living phenomena. This finding is consistent with the idea that the basic mechanism to generate the solar microwave flux is operating at a broad range of time scales as in the case of a turbulence cascade. On the other hand, the time resolution of the presently available data is limited to $0.5 \mathrm{~s}$, which prevents us from studying the range of the possibly elementary time scales. It is important to note that for all observations investigated here conventional methods, such as correlation and spectral analysis, fail to yield this result.

(2) There is no unique well-developed statistical theory for the applied analysis methods yet. To check the reliability of the estimates obtained, we have applied these techniques (power-law section of both structure function and scalegram) also to surrogates chosen to agree in some statistical properties with the data. For solar impulsive mm-wave bursts, the resemblance with the fractional Brownian motion, governed by a stochastic nonlinear evolution equation and characterized by scaling exponents $H>0.5$, which reflect long-range correlations, is evident.

(3) While not distinguishable by the method of power spectrum, the structure function and the multiresolution analysis show clearly different scaling exponents for bursts, the quiet Sun, and the sky background.

Acknowledgements. We acknowledge fruitful discussions with L. Vlahos, A. Benz, and P. Maaß. We thank Ph. Bendjoya and J.-M. Petit for providing the MRA code. The work of B.K. was supported by DARA grant 50QL9208. Finally, we thank the referee for his helpful criticism.

\section{References}

Aschwanden M.J., Schwartz R.A., Alt D.M., 1995, ApJ 447, 932

Bendjoya Ph., Petit J.-M., Spahn F., 1993, ICARUS 105, 385

Benz A.O., 1986, Solar Phys. 104, 99

Benz A.O., 1993, Plasma Astrophysics. Kluwer, Dordrecht

Benz A.O., Aschwanden M.J., 1992, in: Eruptive Solar Flares, Jackson B.V., Machado M.E. and Švestka Z.F. (eds.), Lect. Notes Phys. 399, 106
Benz A.O., Su. H., Magun A., Stehling W., 1992, A\&AS 93, 539

Crosby N.B., Aschwanden M.J., Dennis B.R., 1993, Solar Phys. 143,275

Daubechies I., 1992, Ten Lectures on Wavelets, Society for Industrial and Applied Mathematics, Philadelphia

Feder J., 1988, Fractals. Plenum Press, New York

Flandrin P., 1994, Time-Scale Analysis and Self-Similar Stochastic Processes, Byrnes J.S., Byrnes J.L., Hargreaves K.A. \& Berry K. (eds.): Wavelets and Their Applications, NATO ASI Series C 442. Kluwer Academic Publishers, Dordrecht-Boston-London, pp. 121-142

Güdel M., Benz A.O., 1990, A\&A 231, 202

Isliker H., Kurths J., 1993, Int. J. Bifurcation Chaos 3, 1573

Kiplinger A.L., Dennis B.R., Emslie A.G., Frost K.J., Orwig L.E., 1983, ApJ 265, L99

Kliem B., 1995, in: Coronal Magnetic Energy Releases, Benz A.O. and Krüger A. (eds.), Lect. Notes Phys. 444. Springer, Berlin, p. 93

Krüger A., Aurass H., Kliem B., Urpo S., 1987, in: The Sun, Hejna L. and Sobotka M. (eds.), Publ. Astron. Inst. Czechoslovak. Acad. Sci. 66, 245

Krüger A., Kliem B., Hildebrandt J., Zaitsev V.V., 1994, ApJS 90, 683

Kurths J., Schwarz U., Witt A., 1995, in: Coronal Magnetic Energy Releases, Benz A.O. and Krüger A. (eds.), Lect. Notes Phys. 444. Springer, Berlin, p. 159

Lu E.T., Hamilton R.H., 1991, ApJ 380, L89

Lu E.T., Hamilton R.H., McTiernan J.M., Bromund K.R., 1993, ApJ 412, 841

Mallat S.G., 1989, IEEE Trans. Pattern Anal. Mach. Intell. 11, 674

Masuda S., Kosugi T., Hara H., Tsuneta S., Ogawara Y., 1994, Nat 371,495

Meyer Y., Ryan R.D., 1993, Wavelets. Algorithms and Applications, Society for Industrial and Applied Mathematics, Philadelphia

Osborne A.R., Provenzale A., 1989, Physica D 35, 357

Priestley M.B., 1981, Spectral Analysis and Time Series. Academic Press, London and New York

Scargle J.D., 1993, Wavelet Methods in Astronomical Time Series Analysis, in Lessi O. (ed.) International Conference on Applications of Time Series Analysis in Astronomy and Meteorology, Padua

Scargle J.D., Steinman-Cameron T., Young K., et al., 1993, ApJ 411, L91

Schumacher J., Kliem B., 1996, Phys. Plasmas 3, 4703

Slottje C., 1978, Nat 275, 520

Sturrock P.A., Kaufmann P., Moore R.L., Smith D.F., 1984, Solar Phys. 94, 431

Tajima T., Sakai J., Nakajima H., et al., 1987, ApJ 321, 1031

Tsuneta S., 1996, ApJ 456, 840

Urpo S., Pohjolainen S., Teräsranta H., 1992, Helsinki University of Technology, Metsähovi Radio Research Station Series A, Report 12

Vigouroux A., Delache Ph., 1994, A\&A 278, 607

Vlahos L., Georgoulis M., Kluiving R., Paschos P., 1995, A\&A 299, 897

Zaitsev V.V., Stepanov A.V., 1991, AZh 68, 384

Zaitsev V.V., Stepanov A.V., 1992, Solar Phys. 139, 343 